\title{
Control of an Outbreak of Acinetobacter baumannii in Burn Unit in a Tertiary Care Hospital of North India
}

\author{
Shweta Sharma, Nirmaljit Kaur, Shalini Malhotra, Preeti Madan, and Charoo Hans \\ Department of Microbiology, Dr. Ram Manohar Lohia Hospital \& PGIMER, Baba Kharak Singh Marg, New Delhi 110001, India \\ Correspondence should be addressed to Shweta Sharma; drshwetamicro@gmail.com
}

Received 18 July 2014; Revised 15 September 2014; Accepted 20 September 2014; Published 28 September 2014

Academic Editor: Julio Diaz

Copyright ( 2014 Shweta Sharma et al. This is an open access article distributed under the Creative Commons Attribution License, which permits unrestricted use, distribution, and reproduction in any medium, provided the original work is properly cited.

\begin{abstract}
Acinetobacter infection is increasing in hospitals and now it is considered as a global threat, as it can be easily transmitted and remain viable in the hospital environment for a long time due to its multidrug-resistant status, resistance to desiccation, and tendency to adhere to inanimate surfaces. Outbreaks caused by multidrug-resistant Acinetobacter baumannii (MDRAB) are difficult to control and have substantial morbidity and mortality, especially in vulnerable host. Here we are describing an outbreak of multidrugresistant Acinetobacter baumannii in burn unit of a tertiary care hospital in India followed by its investigation and infection control measures taken to curtail the outbreak. Outbreak investigation and environmental sampling are the key factors which help in deciding the infection control strategies for control of outbreak. Implementation of contact precautions, hand hygiene, personnel protective equipment, environmental disinfection, isolation of patients, and training of health care workers are effective measures to control the outbreak of MDRAB in burn unit.
\end{abstract}

\section{Introduction}

Burn wound surface provides a favourable niche for microbial colonization and proliferation. Burn wound infections may originate from the patient's endogenous skin and gastrointestinal and respiratory flora (endogenous) or may also be transferred via contact with contaminated external surfaces and soiled hands of healthcare workers (exogenous). Burn patients are more susceptible to colonization from organisms in the environment as well as disperse organisms into the surrounding environment [1]. Acinetobacter spp. remain as normal skin flora, can be easily transmitted, and remain viable in the hospital environment for a long time due to its multidrug-resistant status, resistance to desiccation, and tendency to adhere to inanimate surfaces; hence Acinetobacter infection is increasing in hospitals and now it is considered as a global threat. Risk factors associated with Acinetobacter infection include invasive procedures which are commonly required in patients with burn injuries, like mechanical ventilation, central venous or urinary catheters, and broadspectrum antimicrobials [2]. Outbreaks caused by multidrugresistant Acinetobacter baumannii (MDRAB) are difficult to control and have substantial morbidity and mortality, especially in vulnerable host. Here, we are describing an outbreak of multidrug-resistant Acinetobacter baumannii in burn unit of a tertiary care hospital in India, followed by its investigation and infection control measures taken to curtail the outbreak.

\section{Materials and Methods}

Burn unit in our hospital has both burn patients and plastic surgery patients or old burn cases. These are separated and are placed in different cubicles and also the dressing rooms for both the types of patients are different with different nursing staff. A total of 12 beds are allotted for new burn patients, which are distributed in various cubicles according to the severity of burn and condition of the patient. There are 2 beds in one cubicle, 4 beds in the second cubicle, and 6 beds in the third cubicle, with a distance of approximately 10 feet between two beds, with approximate total admissions of 20-25 burn patients in the month. The second cubicle with 4 beds is the intensive care unit with ventilator and cardiac monitor dedicated to all the beds, for critical patients with large burn surface area and infected with multidrug resistant organisms. Burn wound swabs which were received 
in microbiology department were processed according to standard microbiological techniques. MDRAB was defined as $A$. baumannii resistant to three or more antimicrobials of the following: aminoglycoside, $\beta$-lactam- $\beta$-lactamase inhibitor, antipseudomonal carbapenem, antipseudomonal cephalosporin, and fluoroquinolone [3]. For environmental sampling, sterile swabs moistened with sterile distilled water were used to swab items in the burn ward, like dressing room trolley, transport trolley, patient's beds, silver sulfadiazine ointment, water taps, and so forth. The hands of health care workers (HCWs), including burn dressers, doctors, nurses, were also sampled to assess the potential of hand carriage. They were immediately inoculated onto $5 \%$ sheep blood agar plates and in BHI broth and incubated overnight in air at $37^{\circ} \mathrm{C}$. Microorganisms were identified by conventional methods and were tested for susceptibility to amikacin, aztreonam, ceftriaxone, cefotaxime, ciprofloxacin, cotrimoxazole, cefotaxime-clavulanic acid, colistin, gentamicin, imipenem, piperacillin-tazobactam, and tigecycline using Kirby Bauer disc diffusion method according to CLSI guidelines [4]. All the isolates were confirmed and also breakpoint MICs were done with automated system, Microscan WalkAway 40 plus system, as per manufacturer's guidelines.

\section{Outbreak Investigation}

Acinetobacter baumannii was common in causing burn wound infections, but its incidence was 1 to 2 isolates in one to two weeks till March 2014. However the isolation of the organism increased to 1 to 2 cases in two to three days, in April 2014, and Acinetobacter spp. was isolated in total of 12 cases. Acinetobacter baumannii was identified on the basis of various biochemicals like motility, oxidase, OF media, sugars, indole, methyl red, Voges Proskauer, citrate, urease, triple sugar iron agar, $10 \%$ lactose, hemolysis, growth at $44^{\circ} \mathrm{C}$, and so forth. And also the isolates were confirmed as Acinetobacter baumannii in Microscan WalkAway 40 plus system. All the isolates were MDRAB with the same antibiogram, that is, resistant to amikacin $(>32 \mu \mathrm{g} / \mathrm{mL})$, aztreonam $(>16 \mu \mathrm{g} / \mathrm{mL})$, ceftriaxone ( $>32 \mu \mathrm{g} / \mathrm{mL})$, cefotaxime $(>32 \mu \mathrm{g} / \mathrm{mL})$, ciprofloxacin $(>2 \mu \mathrm{g}$ / $\mathrm{mL})$, cotrimoxazole $(>2 / 38 \mu \mathrm{g} / \mathrm{mL})$, cefotaxime-clavulanic acid $(>4 \mu \mathrm{g} / \mathrm{mL})$, gentamicin $(>8 \mu \mathrm{g} / \mathrm{mL})$, and piperacillintazobactam $(>64 \mu \mathrm{g} / \mathrm{mL})$, and were sensitive to imipenem $(\leq 4 \mu \mathrm{g} / \mathrm{mL})$, colistin, and tigecycline by Kirby Bauer disc diffusion method and MICs by Microscan WalkAway 40 plus system. Hence, infection control team was informed about increase in number of cases and environmental samples were taken by infection control nurse especially from the dressing room of burn unit. Overall 18 swabs were collected, from various areas in the burn ward especially from the dressing room like dressing room trolley, transport trolley, patient's beds, silver sulfadiazine ointment, water taps, and so forth. Out of them, two swabs were positive for Acinetobacter baumannii, one collected from the burn dressing trolley and the other from the transport trolley, which was especially used for transport of patient from the bed to the dressing room. Both the isolates from swabs were showing the same sensitivity pattern as the MDRAB isolated from patient's wound swab. All the isolates were confirmed by Microscan automated system. Samples were also collected from the hands of eight health care workers including two burn dressers, and none of them were positive for Acinetobacter baumannii.

3.1. Outbreak Control Measures. The infection control team established the outbreak control strategies which were implemented in May 2014. All health care staff including dressers and nursing staff were taught probable routes of transmission, with emphasis on patient to patient cross-contamination and infection control measures such as hand hygiene, standard precautions, aseptic techniques, personnel protective equipment, environmental cleaning and disinfection, and also biomedical waste management. Proper steps of hand hygiene were demonstrated to health care workers and also the posters of hand washing were placed in various areas of the burn ward. Also the use of alcohol based hand rubs with automatic dispensers was reinforced in the ward so that hand hygiene could be improved before and after contact with the patient. The use of gloves wherever necessary was emphasised. Staff allocated for affected patients was not allowed to care for any unaffected patients, till three negative cultures were obtained from affected patient at weekly intervals. Burn dressers used sterile gloves, mask, and gowns for dressing of burn patients and gown and gloves were changed after every patient to be disinfected later, and hands were washed before and after removing gloves and wearing another glove in between each patient. Burn wounds were occlusively wrapped and never kept open to prevent crosscontamination of environment. All the bandages and dressing material used for dressing were preautoclaved; local agent like silver sulfadiazine was applied aseptically and separate nursing staff was provided to guide the dressers and for support during dressing. Dressing frequency was increased to be daily if total burn surface area was $>35-40 \%$ or if the dressings were soaked; otherwise with lesser burn surface area dressing was done on alternate days. Dressing rooms were disinfected with high level disinfectants like hydrogen peroxide with silver nitrate, which is dispersed with the help of a fogger-like device inside the dressing room for a contact time of 1 hour, before starting the dressings. Patients were shifted to dressing room in transport trolley and then shifted to dressing trolley which was thoroughly disinfected between each patient. The bed of the patient and the trolley of patient were disinfected regularly and the bed sheets for each patient were changed daily. In case of discharge or death of the patient, the area was cleaned and disinfected thoroughly before taking any new patient. The frequency of environmental cleaning and disinfection was increased to 3-4 times in a day, and hydrogen peroxide with silver nitrate was used for disinfection. Entry of visitors was limited in the ward, and only one person was allowed with each patient and shoe covers were made compulsory for entry in the ward. Patient relatives were also trained by nursing staff regarding infection control measures to be taken to control the spread of infection. Proper disposal of soiled linen and other biomedical waste was undertaken in the ward. Antibiotic usage was limited to short periods, and ceftazidime 
and amikacin were used empirically, and other antibiotic usage is according to the results of cultures and antibiotic susceptibility report. MDRAB patients were switched to carbapenems, as they were susceptible to imipenem.

3.2. Results of Outbreak Control Measures. After the introduction of infection control measures, the number of newly diagnosed cases began to decline by the end of May, and June 2014, to 1-2 Acinetobacter baumannii in two weeks. Also the environmental samples collected from the dressing room, dressing trolley, transport trolley, and so forth stopped growing Acinetobacter baumannii.

\section{Discussion}

An outbreak of MDR organism occurs in a particular unit due to lapses in infection control measures, resulting in an increased cross-transmission between patients [5]. The outbreak reported here appeared to be initiated from the dressing trolley or transport trolley, because the sensitivity pattern was exactly the same in the isolates cultured from these areas and from patient's burn wound swabs. Also the enhancement and appropriate implementation of barrier precautions, hand hygiene, and disinfection at the time of dressing of patients were found very effective in preventing the spread of MDRAB. Environmental sampling was found effective to find the source of spread of MDRAB in the ward and thus helped to make the outbreak control strategies in our study and to guide the cleaning and disinfection of environmental sites and to check the quality of environmental cleaning after thorough disinfection of environment. Many previous studies have reported isolation of Acinetobacter species from environmental sites and hence environmental cleaning is a major part of infection control strategy [6-8]. However, it is difficult to curtail the outbreak of multidrug resistant organisms by merely environmental disinfection especially in the burn unit as these patients are more susceptible to infections due to loss of normal skin barrier and large colonised burn area can easily spread the MDR strains in the environment and also to other patients due to cross-contamination. Hence isolation of patients and contact precautions along with proper disposal of soiled linen and education of patient's relatives and dressers are equally important for the control of outbreak as seen in our study [9].

The outbreak in our burn unit was controlled after implementation of various measures, and it is not possible to describe which measure ultimately controlled the outbreak; however, control of environmental reservoir by disinfection and barrier precautions at the time of dressing of patients with education of dressers were considered as the major factors in controlling the outbreak. In some previous studies, complete closure of units was necessary to control outbreaks [10]. However, it was not done in our hospital as it is a very renowned tertiary care centre for burn patients with a load of referrals, and still the outbreak was controlled. Hence strict precautions and environmental disinfection may control the outbreak without closure of the unit. The limitation of this study was that the molecular typing of strains by pulsed field gel electrophoresis (PFGE) was not done in our study; hence it is difficult to say that the outbreak was caused by a single clone. However, the sensitivity pattern was the same in the isolates from environment and from patients, suggesting the possibility of transmission of a single clone responsible for this outbreak.

To conclude, this study shows that outbreak investigation and environmental sampling are the key factors which help in deciding the infection control strategies for control of outbreak. Implementation of contact precautions, hand hygiene, personnel protective equipment, environmental disinfection, isolation of patients, and training of health care workers as well as patient's attendants are effective measures to control the outbreak of MDRAB in burn unit.

\section{Conflict of Interests}

The authors declare that there is no conflict of interests regarding the publication of this paper.

\section{References}

[1] S. Erol, U. Altoparlak, M. N. Akcay, F. Celebi, and M. Parlak, "Changes of microbial flora and wound colonization in burned patients," Burns, vol. 30, no. 4, pp. 357-361, 2004.

[2] K. J. Towner, "Acinetobacter: an old friend, but a new enemy," Journal of Hospital Infection, vol. 73, no. 4, pp. 355-363, 2009.

[3] WHO, "Multidrug-resistant Acinetobacter baumannii (MDRAB)," Fact Sheet 1, World Health Organisation, Metro Manila, Philippines, 2010.

[4] Clinical and Laboratory Standards Institute, "Performance standards for antimicrobial susceptibility testing," TwentyThird Informational Supplement M100-S23, 2013.

[5] E. M. C. D’Agata, V. Thayer, and W. Schaffner, "An outbreak of Acinetobacter baumannii: the importance of crosstransmission," Infection Control and Hospital Epidemiology, vol. 21, no. 9, pp. 588-591, 2000.

[6] M. Denton, M. H. Wilcox, P. Parnell et al., "Role of environmental cleaning in controlling an outbreak of Acinetobacter baumannii on a neurosurgical intensive care unit," Journal of Hospital Infection, vol. 56, no. 2, pp. 106-110, 2004.

[7] M. E. Falagas and P. Kopterides, "Risk factors for the isolation of multi-drug-resistant Acinetobacter baumannii and Pseudomonas aeruginosa: a systematic review of the literature," Journal of Hospital Infection, vol. 64, no. 1, pp. 7-15, 2006.

[8] C. Landelle, P. Legrand, P. Lesprit et al., "Protracted outbreak of multidrug-resistant Acinetobacter baumannii after intercontinental transfer of colonized patients," Infection Control and Hospital Epidemiology, vol. 34, no. 2, pp. 119-124, 2013.

[9] J. D. Naranjo, J. I. V. Navarro, M. S. Busselo et al., "Control of a clonal outbreak of multidrug-resistant Acinetobacter baumannii in a hospital of the Basque country after the introduction of environmental cleaning led by the systematic sampling from environmental objects," Interdisciplinary Perspectives on Infectious Diseases, vol. 2013, Article ID 582831, 9 pages, 2013.

[10] A. Markogiannakis, G. Fildisis, S. Tsiplakou et al., "Crosstransmission of multidrug-resistant Acinetobacter baumannii clonal strains causing episodes of sepsis in a trauma intensive care unit," Infection Control and Hospital Epidemiology, vol. 29, no. 5, pp. 410-417, 2008. 


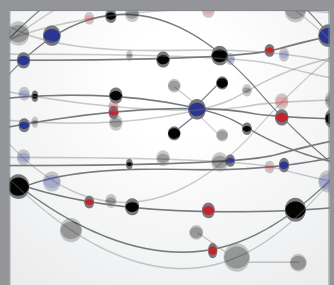

The Scientific World Journal
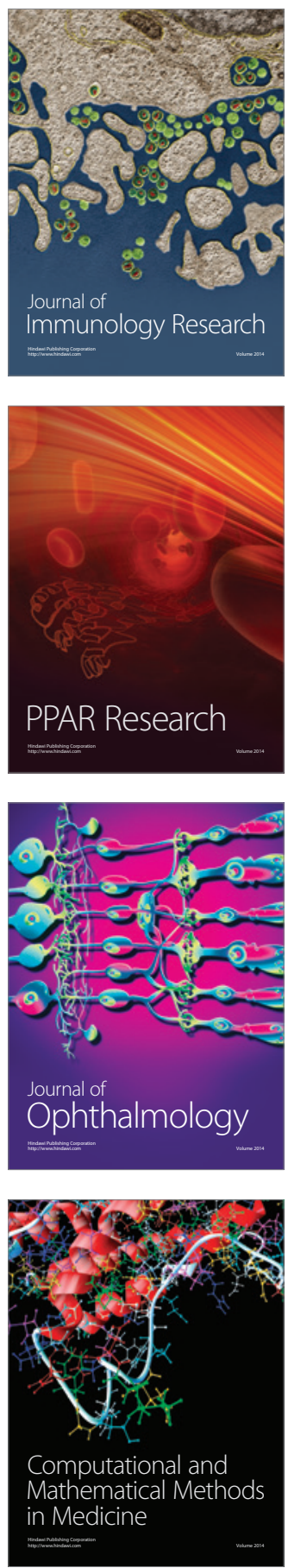

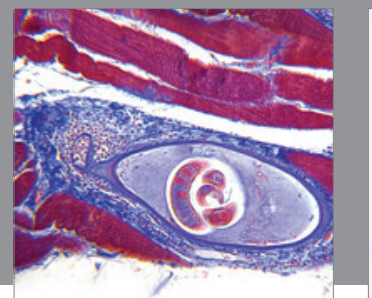

Gastroenterology

Research and Practice
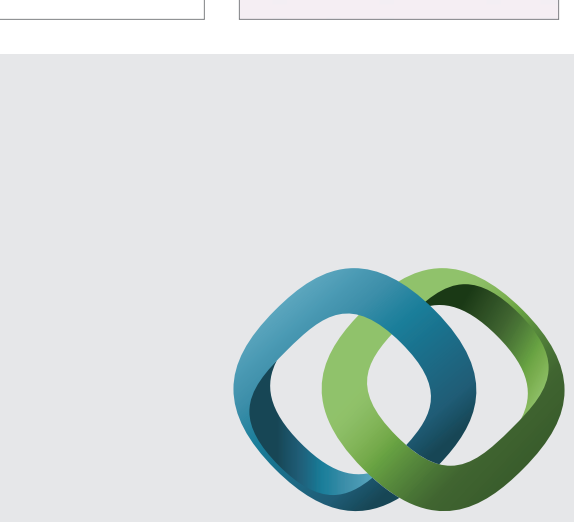

\section{Hindawi}

Submit your manuscripts at

http://www.hindawi.com
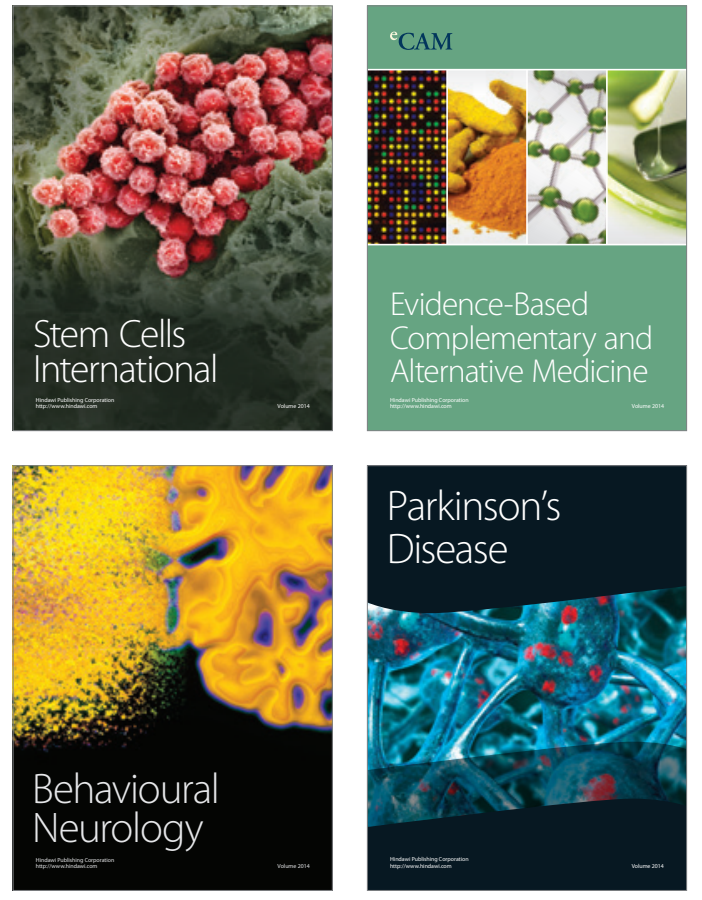
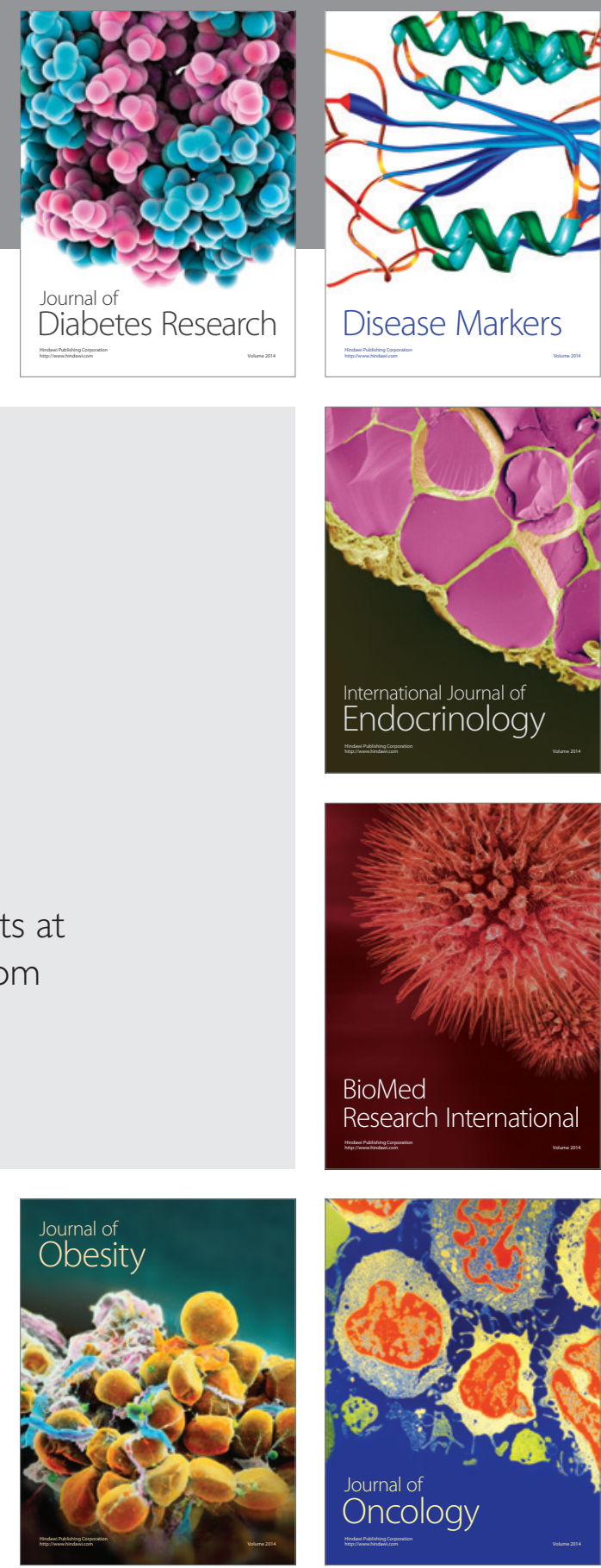

Disease Markers
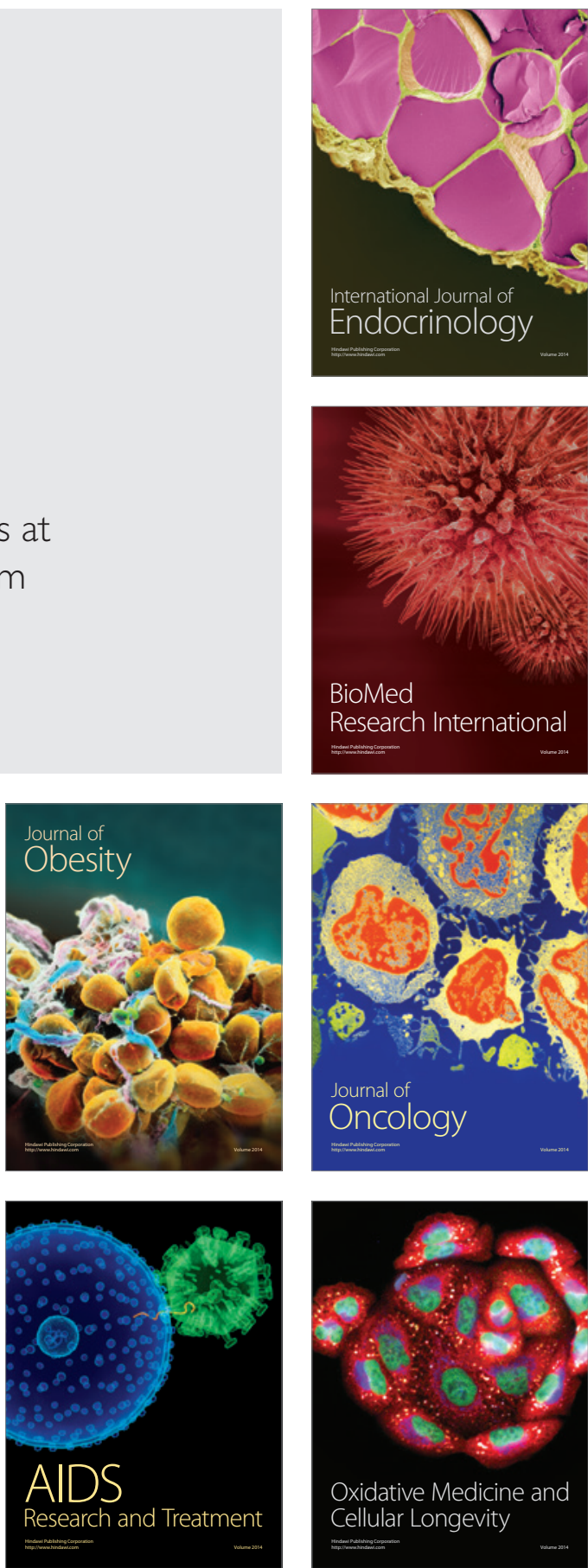